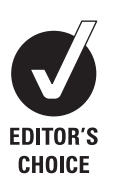

CHOICE

\title{
Exercise during pregnancy improves maternal glucose screen at 24-28 weeks: a randomised controlled trial
}

\author{
Ruben Barakat, ${ }^{1}$ Yaiza Cordero, ${ }^{1}$ Javier Coteron, ${ }^{1}$ Maria Luaces, ${ }^{2}$ Rocio Montejo ${ }^{2}$
}

${ }^{1}$ Facultad de Ciencias de la Actividad Física y del Deporte INEF, Universidad Politécnica de Madrid, Spain

${ }^{2}$ Department of Obstetric and Gynecology, Hospital de Fuenlabrada, Madrid, Spain

\section{Correspondence to} Ruben Barakat, Facultad de Ciencias de la Actividad Física y del Deporte - INEF, Universidad Politécnica de Madrid, Martín Fierro, 28040 Madrid, Spain;

barakatruben@gmail.com

Accepted 30 August 2011 Published Online First 26 September 2011

\section{ABSTRACT}

Objective The influence of an exercise programme performed by healthy pregnant women on maternal glucose tolerance was studied.

Study design A physical activity (PA, land/aquatic activities) programme during the entire pregnancy (three sessions per week) was conducted by a qualified instructor. 83 healthy pregnant women were randomly assigned to either an exercise group ( $E G, n=40$ ) or a control (CG, $n=43$ ) group. $50 \mathrm{~g}$ maternal glucose screen (MGS), maternal weight gain and several pregnancy outcomes were recorded.

Results Significant differences were found between study groups on the $50 \mathrm{~g}$ MGS. Values corresponding to the EG $(103.8 \pm 20.4 \mathrm{mg} / \mathrm{dl})$ were better than those of the CG $(126.9 \pm 29.5 \mathrm{mg} / \mathrm{dl}), \mathrm{p}=0.000$. In addition, no differences in maternal weight gain and no cases of gestational diabetes in EG versus 3 in CG (7\%) $(p>0.05)$ were found.

Conclusion A moderate PA programme performed during pregnancy improves levels of maternal glucose tolerance.

\section{INTRODUCTION}

The prevalence estimate of gestational diabetes mellitus (GDM), which is a disorder of glucose metabolism, varies; however, GDM complicates approximately $4-12 \%$ of pregnancies and is associated with long and short-term morbidity in both the offspring and mother. Adverse infant outcomes include macrosomia, hypoglycaemia, erythemia, hypocalcaemia, jaundice and birth trauma. ${ }^{12}$

Later in life, these children are more likely to become obese, have an abnormal glucose tolerance and develop diabetes in adolescence or early adulthood, when compared with the offspring of normoglycaemic women. ${ }^{3}$

Women diagnosed with GDM are at a high risk for future diabetes mellitus (DM), with approximately $50 \%$ of women developing type 2 diabetes within 5 years of delivery. They are also more likely to display features of insulin resistance syndrome, which are linked to cardiovascular disease. $^{45}$

In recent years, many studies have revealed that antepartum $50 \mathrm{~g}$ maternal glucose screen (50 g MGS), a standard element of current obstetrical care instituted for the purpose of detecting GDM, may provide a previously unrecognised insight into a woman's future risk of metabolic and vascular disease. ${ }^{67}$
The American Diabetes Association in its more recent position statement suggests that all pregnant women should be screened for GDM between the 24 th and 28th week of gestation, unless they are of low-risk status. Two approaches have been suggested for screening of GDM (at 24-28 weeks). ${ }^{8}$

The results of the HAPO study group indicate strong, continuous associations of maternal glucose levels below those diagnostic of GDM and show the risks of adverse outcomes associated with various degrees of maternal glucose intolerance during pregnancy. ${ }^{9} 10$

With the rising incidence of GDM across the developed world, largely paralleling the increased prevalence of obesity, there has been a sharp increase in the risk of pregnancy complications developing related to the birth of macrosomic babies.

Important risk factors of GDM such as high maternal age, family history of type 2 DM, overweight before pregnancy and glucose intolerance during pregnancy are present. There is also recent scientific evidence that excessive maternal weight gain especially early in pregnancy may increase the risk of GDM. ${ }^{11-14}$ Medical advice recommends that normal weight women (body mass index (BMI) $19.8-26.0 \mathrm{~kg} / \mathrm{m}^{2}$ ) gain $11.4-15.9 \mathrm{~kg}$ during pregnancy and overweight women (BMI 26.1-29.0 $\mathrm{kg} / \mathrm{m}^{2}$ ) gain $6.8-11.4 \mathrm{~kg} .{ }^{13}$

Weight gains within these guidelines are associated with healthy fetal and maternal outcomes; weight gains below these goals are associated with low infant birth weight and higher weight gains are associated with macrosomia in the infant. Moreover, women who gain more-thanrecommended weight retain twice as much weight after pregnancy as women who gain within the recommendations. ${ }^{15}$

Physical activity (PA) may contribute to the prevention of excessive maternal weight gain, and thus is crucial for dissecting the vicious circle involving GDM, childhood obesity and adulthood obesity and diabetes. ${ }^{16-18}$ In Mottola's opinion, the true effectiveness of specific structured exercise programmes remains untapped in GDM prevention. $^{7}$

Indeed, the benefits of physical training are discussed along with recommendations for varying activities, PA-associated blood glucose management, diabetes prevention, gestational diabetes, and safe and effective practices for PA with diabetes-related complications. ${ }^{19}$

Current evidence suggests that both diet and exercise can alter the usual increase in insulin resistance seen in Western societies during mid 
and late pregnancy. ${ }^{20}$ Specifically, many studies have demonstrated that moderate PA during pregnancy is safe and associated with normal pregnancy outcome. ${ }^{21-23}$

The aim of the present study was to assess the influence of a physical activity program with land/aquatic activities during pregnancy on the $50 \mathrm{~g}$ MGS at 24-28 week, total maternal weight gain and cases of GDM. We hypothesised that regular aerobic exercise during pregnancy would lead to an improved maternal glucose tolerance and be associated with a lower maternal weight gain and fewer cases of GDM.

\section{MATERIAL AND METHODS}

The present study was a randomised controlled clinical trial (RCT) in accordance with the recently published CONSORT guidelines (http://www.consort-statement.org) ${ }^{24}$. A flow diagram of the study participants is shown in figure 1.

We studied 83 Caucasian women belonging to a low to medium socioeconomic class. The research protocol was reviewed and approved by the ethical committee and the ethical guidelines of the Declaration of Helsinki, last modified in 2008, were followed. The research was carried out in collaboration with the Obstetric Hospital Department (Centro de Salud de Torrelodones, Madrid, Spain). All participants were informed about the aim and study protocol, and written informed consent was provided by all women.

\section{Subjects of the study}

We recruited 100 pregnant women into the study; after possible adverse effects of training had been studied, we randomly assigned 83 healthy gravida (aged $32 \pm 4$ years) either to an exercise group ( $E G, n=40$ ) or a control (CG, $n=43$ ) group. Women not planning to give birth in the same hospital and not being under medical follow-up throughout the entire pregnancy period were not included in the study. All the women were healthy and had uncomplicated and singleton pregnancies.

The exclusion criteria were any type of absolute obstetric contraindication ${ }^{25}$ such as

Flow Diagram of study participants

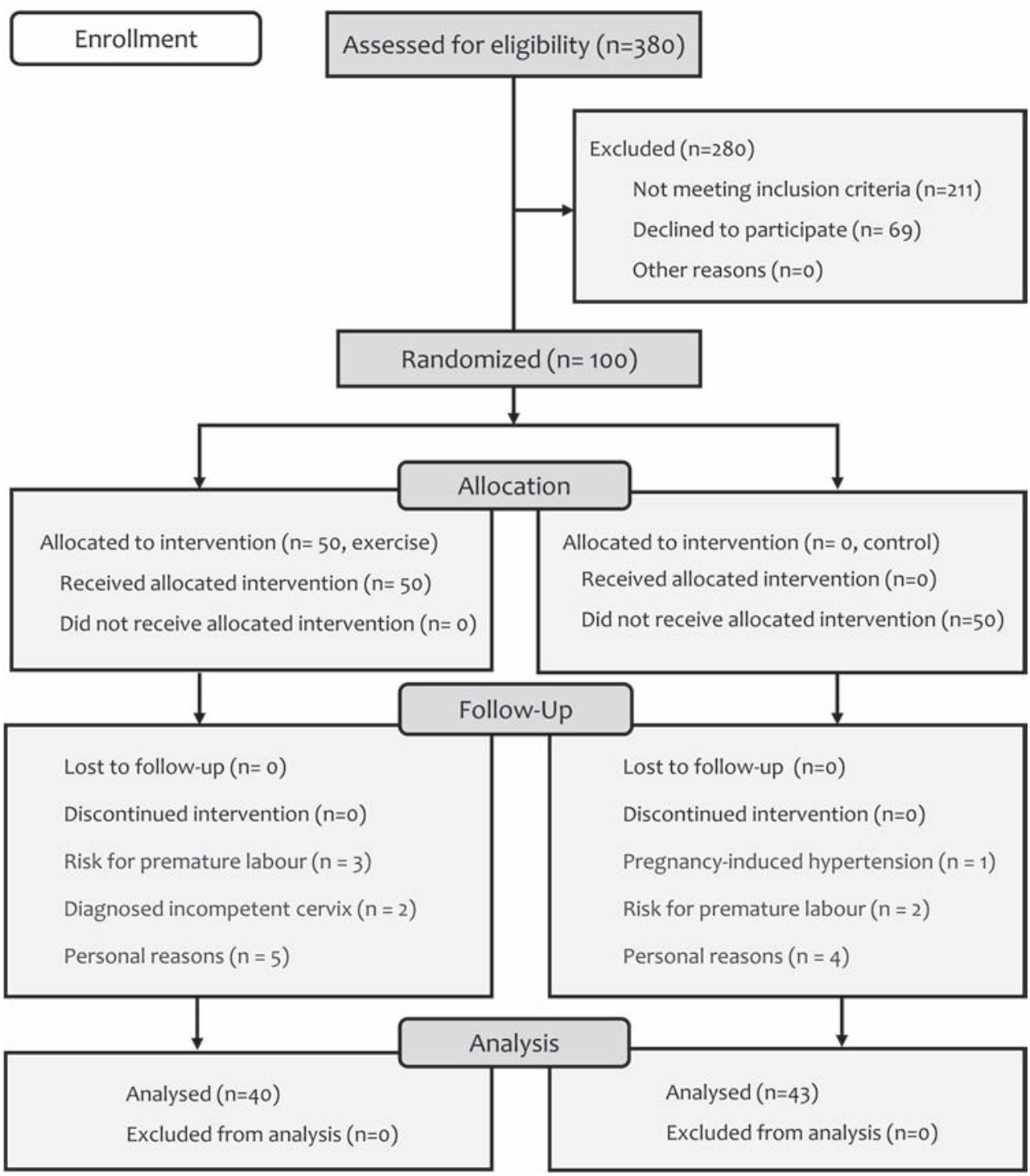

Figure 1 CONSORT 2010 flow diagram of the study participants. 
- Active illness of the myocardium.

- Heart insufficiency.

- Rheumatic heart illness (type II or above).

- Thromboflebitis.

- Recent pulmonary embolism (last 5 years).

- Acquired infectious disease.

- Cervical incompetence.

- Multiple pregnancy.

- Genital haemorrhage.

- Premature breakage of the ovular membranes.

- Retarded interuterine development.

- Fetal macrosomia.

- Serious blood disease.

- Serious hypertension.

- Absence of prenatal control.

- Suspects of fetal suffering.

- Risk of premature labour.

- Prepregnant type 1 or 2 DM.

\section{Physical activity programme}

The physical conditioning programme was a $35-45$-min session performed three times a week (Monday, Wednesday, Friday), with two land aerobic sessions and one aquatic activities session. This was conducted from the start of the pregnancy (weeks 6-9) to the end of the third trimester (weeks 38-39). Thus, an average of 85 training sessions was originally planned for each participant in the event of no preterm delivery. All subjects used a heart rate (HR) monitor (Accurex Plus; Polar Electro OY, Kempele, Finland) during the training sessions to ensure that exercise intensity was light to moderate, that is, their HR was consistently under $70 \%$ of their agepredicted maximum HR value (220 minus age).

\section{Land sessions}

Each session included a 25 -min core session, which was preceded and followed by a gradual warm-up and cool down period (both of 7-8 min duration and consisting of walking and light, static stretching (avoiding muscle pain) of most muscle groups (upper and lower limbs, neck and trunk muscles)). The cool down period also included relaxation and pelvic floor exercises. The core session included the following toning and very light resistance exercises: toning and joint mobilisation exercises including shoulder shrugs and rotations, arm elevations, leg lateral elevations, pelvic tilts and rocks. Resistance exercises were performed through the full range of motion normally associated with correct technique for each exercise and engaged the major muscle groups (pectoral, dorsal, shoulder, upper and lower limb muscles). They included one set (10-12 repetitions of each) of (1) abdominal curls and (2) the following exercises using barbells ( $3 \mathrm{~kg} /$ exercise) or low-to-medium resistance bands (Therabands). We specifically avoided any exercise that involved extreme stretching and joint overextension, ballistic movements or jumps. Any type of exercise on the back was not performed for more than 2 min.

We used exercises that covered the major muscle groups of the arms and abdomen to promote good posture, prevent low back pain and (third trimester) strengthen the muscles of labour and pelvic floor. We have also included in the programme one set of aerobic dance every 2 weeks.

In order to maximise safety, adherence to the training programme and its efficacy, all sessions were (1) supervised by a qualified fitness specialist (working with groups of 10-12 subjects) and with an obstetrician assistance; (2) accompanied by music; and (3) performed in the Health Care Center, in a spacious, well-lit room under favourable environmental conditions (altitude $600 \mathrm{~m}$; temperature $=19-21^{\circ} \mathrm{C}$; humidity $=50-60 \%$ ). More details of the exercise training protocol have been described by Barakat et al. ${ }^{26}$

\section{Aquatic activities}

The main purpose of exercise in the water was to avoid a huge impact. Aquatic sessions include swimming laps, jogging, walking, walking back and forth across the pool, stretching, lunges, step climbs, cross-country skiing movements and strength exercises in the water. Aquatic materials like noodles, floats and light-resistance gloves were also used in the water for muscle conditioning. Water temperature was $28-29^{\circ} \mathrm{C}$ and special prevention considerations to avoid infections were taken into account.

\section{General considerations to exercise}

An adequate intake of calories and nutrients was assured before exercise. As a general rule and to avoid potential risks, the following were avoided:

Activities that included Valsalva's mechanism.

High room temperatures or humid environments, with the aim of avoiding chances of hyperthermia (body temperature more than $38^{\circ} \mathrm{C}$ ).

Ballistic movements (except in water activities).

Positions of extreme muscular tension.

\section{Variables of the study}

Main variables of the study were $50 \mathrm{~g}$ MGS, maternal weight gain and cases of GDM. In the study population, women were routinely screened for gestational diabetes at 24-28 weeks of gestation with a non-fasting oral glucose challenge test in which venous blood was sampled $1 \mathrm{~h}$ after a $50 \mathrm{~g}$ oral glucose load. If the $1 \mathrm{~h}$ glucose result was at least $140 \mathrm{mg} / \mathrm{dl}$, the participant was referred for a $100 \mathrm{~g}$ fasting glucose 3 -h tolerance test. Normal results were a blood glucose below $95 \mathrm{mg} / \mathrm{dl}$ at baseline, below $180 \mathrm{mg} / \mathrm{dl}$ at $1 \mathrm{~h}$, below $155 \mathrm{mg} / \mathrm{dl}$ at $2 \mathrm{~h}$ and below $140 \mathrm{mg} / \mathrm{dl}$ at 3 h. ${ }^{4} 13$

We categorised participants with a normal screening glucose challenge as having normal glucose tolerance and those who failed the challenge test as having abnormal glucose tolerance. We classified those with at least two abnormal results on the fasting glucose tolerance test as having GDM.

Maternal characteristics and other pregnancy outcomes considered (by interview and medical information) were as follows: maternal age, BMI, smoking habits, alcohol intake, occupational activity, time standing per day, time of domestic task, educational level, parity, gestational age, type of delivery, blood pressure, birth weight and Apgar score.

\section{Data analysis}

Our data (presented in tables 1-3) were analysed using the Student's $t$ test for independent samples, one-way ANOVA and $\chi^{2}$ test.

We present maternal characteristics of the study sample by group (EG and CG) in terms of mean and SD, unless otherwise stated. Relative to maternal perception of health status and lacerations type, $\chi^{2}$ tests were used. For group comparisons of pregnancy outcomes, we analysed continuous and nominal data with $t$ test for unpaired data and $\chi^{2}$ tests, respectively. We compared Apgar scores between groups using the non-parametric Mann-Whitney U test.

Statistical analysis was performed with the Statistical Package for Social Sciences software (version 14.0 for Windows; 
Table 1 Maternal characteristics

\begin{tabular}{|c|c|c|c|}
\hline & $E G(n=40)$ & CG $(n=43)$ & p value \\
\hline \multicolumn{4}{|l|}{ Maternal characteristics } \\
\hline Maternal age (year) & $32 \pm 4$ & $31 \pm 3$ & $>0.05$ \\
\hline Body mass index before pregnancy $\left(\mathrm{kg} / \mathrm{m}^{2}\right)$ & $22.7 \pm 2.8$ & $23 \pm 2.9$ & $>0.05$ \\
\hline \multicolumn{4}{|l|}{ Smoking habits $(\mathrm{n} / \%)$} \\
\hline Yes & $2 / 5$ & $3 / 7$ & \multirow{3}{*}{$>0.05$} \\
\hline No & $27 / 67.5$ & $27 / 62.8$ & \\
\hline Before pregnancy, not now & $11 / 27.5$ & $13 / 30.2$ & \\
\hline \multicolumn{4}{|l|}{ Alcohol intake (n/\%) } \\
\hline Yes & $2 / 5$ & $3 / 7$ & \multirow{2}{*}{$>0.05$} \\
\hline No & $38 / 95$ & $40 / 93$ & \\
\hline \multicolumn{4}{|l|}{ Occupational activity (n/\%) } \\
\hline Sedentary job & $21 / 52.5$ & $23 / 53.5$ & \multirow{3}{*}{$>0.05$} \\
\hline Housewife & $4 / 10$ & $7 / 16.3$ & \\
\hline Active job & $15 / 37.5$ & $13 / 30.2$ & \\
\hline \multicolumn{4}{|l|}{ Hours standing $(n / \%)$} \\
\hline$>3 \mathrm{~h}$ & $18 / 45$ & $17 / 39.5$ & \multirow{2}{*}{$>0.05$} \\
\hline$<3 \mathrm{~h}$ & $22 / 55$ & $26 / 60.5$ & \\
\hline \multicolumn{4}{|l|}{ Maternal education $(\mathrm{n} / \%)$} \\
\hline$<$ High school & $0 / 0$ & $16 / 37.2$ & \\
\hline High school & $7 / 17.5$ & $21 / 48.8$ & \multirow[t]{2}{*}{$<0.05$} \\
\hline$>$ High school & $33 / 82.5$ & $6 / 14$ & \\
\hline \multicolumn{4}{|l|}{ Parity } \\
\hline 0 gestation before & $26 / 65$ & $21 / 48.8$ & \multirow{3}{*}{$>0.05$} \\
\hline 1 gestation before & $11 / 27.5$ & $18 / 41.9$ & \\
\hline$>1$ gestation before & $3 / 7.5$ & $4 / 9.3$ & \\
\hline \multicolumn{4}{|l|}{ Exercise habits before gestation } \\
\hline Sedentary & $8 / 20$ & $14 / 32.6$ & \multirow{4}{*}{$>0.05$} \\
\hline Some active & $9 / 22.5$ & $12 / 27.9$ & \\
\hline Active & $17 / 42.5$ & $13 / 30.2$ & \\
\hline Very active & $6 / 15$ & $4 / 9.3$ & \\
\hline
\end{tabular}

Data are expressed as mean $\pm S D$, unless otherwise indicated.

CG, control group; EG, exercise group.

Table $250 \mathrm{~g} \mathrm{MGS,} \mathrm{maternal} \mathrm{weight} \mathrm{gain,} \mathrm{abnormal} 50 \mathrm{~g} \mathrm{MGS}$ and cases of GDM

\begin{tabular}{lllc}
\hline & EG $(\mathbf{n}=\mathbf{4 0})$ & CG $(\mathbf{n}=\mathbf{4 3})$ & p value \\
\hline $50 \mathrm{~g} \mathrm{MGS}(\mathrm{mg} / \mathrm{dl})$ & $103.82 \pm 20.4$ & $126.93 \pm 29.5$ & 0.00 \\
$\begin{array}{l}\text { Maternal weight gain during } \\
\text { pregnancy }(\mathrm{kg})\end{array}$ & $12.5 \pm 3.2$ & $13.8 \pm 3.1$ & $>0.05$ \\
$\begin{array}{lll}\text { Altered } 50 \mathrm{~g} \mathrm{MGS}(\mathrm{n} / \%) \\
\text { Cases of GDM }(\mathrm{n} / \%)\end{array}$ & $2 / 5$ & $5 / 11.5$ & $>0.05$ \\
\hline
\end{tabular}

CG, control group; EG, exercise group; GDM, gestational diabetes mellitus; MGS, maternal glucose screen.

SPSS Inc, Chicago, Illinois, USA) and the level of significance was set to $<0.05$.

\section{RESULTS}

Our results are presented in table 1 (maternal characteristics), table 2 (50 g MGS, altered $50 \mathrm{~g}$ MGS, maternal weight gain and cases of GDM) and table 3 (pregnancy outcomes).

\section{Adherence to training and possible adverse effects}

According to our analysis and with regard to adherence to training and its possible adverse effects, we report the following: 10 women from the EG discontinued the intervention because of risk of premature labour $(n=3)$, incompetent cervix was diagnosed $(n=2)$ and personal reasons such as change of residence
Table 3 Pregnancy outcome

\begin{tabular}{lccc}
\hline & EG $(\mathbf{n}=\mathbf{4 0})$ & $\mathbf{C G}(\mathbf{n}=\mathbf{4 3})$ & $\mathbf{p}$ value \\
\hline Gestational age (days) & $277.1 \pm 9.2$ & $278.2 \pm 7.5$ & $>0.05$ \\
Type of delivery & & & \\
$\quad$ Normal (n/\%) & $23 / 57.5$ & $26 / 60.5$ & $>0.05$ \\
Instrumental (n/\%) & $5 / 12.5$ & $11 / 25.6$ & \\
$\quad$ Caesarean (n/\%) & $12 / 30$ & $6 / 14$ & \\
Systolic blood pressure (mm Hg) & $109.8 \pm 10.7$ & $114.3 \pm 10.4$ & $>0.05$ \\
Diastolic blood pressure (mm Hg) & $65.1 \pm 8.1$ & $67.4 \pm 8.9$ & \\
Newborn characteristics & & & \\
Birth weight (g) & $3404 \pm 465$ & $3465 \pm 411$ & $>0.05$ \\
Apgar score 1 min & $8.7 \pm 1.1$ & $8.7 \pm 0.8$ & $>0.05$ \\
Apgar score 5 min & $9.9 \pm 0.9$ & $9.9 \pm 0.7$ & $>0.05$ \\
Size of newborn & $50.07 \pm 2.4$ & $49.95 \pm 1.9$ & $>0.05$ \\
\hline
\end{tabular}

CG, control group; EG, exercise group.

$(n=5)$. Seven participants of the CG were excluded from the study because of pregnancy-induced hypertension $(n=1)$, risk for premature labour $(n=2)$ and personal reasons $(n=4)$. The final number of participants included were 40 in the EG and 43 in the CG. No exercise-related injuries were experienced during pregnancy. Adherence to training in the experimental group was $85 \%$. No women changed from the CG to the EG or vice versa and there were no protocol deviations from study as planned.

\section{Maternal characteristics}

At the beginning of our study, no between-group significant differences $(p>0.05)$ were found with regard to the potential confounding variables such as occupational activities, standing, smoking habits and alcohol intake that could potentially have an influence on the main variables of the study, except level of maternal education $(p<0.05)$.

\section{Main variables of study}

Corresponding values to $50 \mathrm{~g}$ MGS also present minor values in EG $(126.9 \pm 29.5 \mathrm{mg} / \mathrm{dl})$ than in CG $(103.8 \pm 20.4 \mathrm{mg} / \mathrm{dl})$ $(p<0.05)$. Relative to women with altered values on the first step 50 g MGS (CG=5, EG=2), three women were diagnosed of GDM in CG, while no cases of GDM were reported in EG. There was no difference between the groups with regard to total maternal weight gain ( $p>0.05)$.

\section{Pregnancy outcome}

As shown in table 3 , we observed that other pregnancy outcomes did not differ between the two groups $(p>0.05)$. There have been no cases of fetal macrosomia in both study groups; we found one newborn with Apgar score at $5 \mathrm{~min}<7$ in EG.

We analysed continuous and nominal data with $t$ test for unpaired data and $\chi^{2}$ tests, respectively, at the level of $p=0.05$.

\section{Comment}

This trial is expected to be effective in the short-term prevention of abnormal $50 \mathrm{~g}$ MGS. We also believe that this might act as a factor for the prevention of excessive maternal weight gain and GDM.

Moreover, we did not observe any adverse effect on pregnancy outcome, except type of delivery, with the caesarean section rate being double in the EG $(12 / 30 \%$ vs $6 / 14 \%)$. This could be because of an increased number of primiparous women in the EG, and these women have more complications during delivery, especially in the first stage of labour. ${ }^{27}$ 
A controversy exists relative to the influence of exercise on the type of delivery; some authors reported an increased caesarean section rate in EG, ${ }^{28-30}$ while other studies informed no influence of PA on type of delivery. ${ }^{22} 3132$

A novelty (and strength) of this study is the possibility of integrating water and land activities in an exercise programme for pregnant women. The presence of obstetricians and those qualified in sports science for guidance and constant supervision of the programme are additional strengths of our study.

There is increasing evidence of the beneficial effects that PA has on the prevention of excessive maternal weight gain or metabolic disorders during pregnancy.

The results of previous studies report a protective effect of PA against the excessive maternal weight gain, development of GDM during pregnancy or other physiological parameters (ie, glycaemic control, stroke volume). Taken together, results from observational studies and clinical trials suggest that PA may be an important component of prevention and control to excessive maternal weight gain and metabolic disorders. ${ }^{3}$

Barros et al informed of an adequate glycaemic control of pregnant women with GDM by means of a resistance exercise programme. They reported that a significant reduction in the number of patients who required insulin was observed in the EG compared with the CG. ${ }^{33}$

Jovanovic-Peterson et al reported that when those receiving an exercise therapy were compared with those receiving a standard dietary intervention, the former were found to have greater glycaemic control, lower fasting, postprandial glucose concentrations and improved cardiorespiratory fitness. ${ }^{34} 35$

Other studies with different exercise prescriptions (eg, walking programme, $1 \mathrm{~h}$ self-paced bout of walking following a meal, two 30-min sessions on the cycle ergometer) have yielded similar results that document the potential therapeutic benefits of exercise in pregnancy as a way to mitigate the metabolic abnormalities. ${ }^{4} 812$

Oken et al assessed the duration and intensity of PA and time spent viewing television both before and during pregnancy among 1.805 women (cohort study). They found that women who engaged in any vigorous pregestational PA experienced a reduced risk of GDM and abnormal glucose tolerance (OR 0.76, $95 \%$ CI 0.57 to 1.00 ). Women who reported vigorous activity before pregnancy and light-to-moderate or vigorous activity during pregnancy appeared to have a lower risk of both GDM and abnormal glucose tolerance compared with women not reporting these activities in either time period. ${ }^{12}$

Dempsey et $\mathrm{al}^{17}$ in a study of 155 GDM cases and 386 normotensive, non-diabetic pregnant controls found that recreational PA performed before and/or during pregnancy is associated with a reduced risk of GDM.

In contrast to these findings, two therapeutic studies have reported no differences in glycaemic control following exercise. $^{3637}$ In addition, Dye et $a^{\beta 8}$ and Bartollotto et a ${ }^{\beta 9}$ in two different studies did not observe any overall benefit of exercise during pregnancy on glucose tolerance.

Callaway et al examined the feasibility of an individualised exercise programme to prevent GDM in obese pregnant women. They informed that the intervention was feasible and prompted a modest increase in PA. However, they are not confident that this intervention would be sufficient to prevent GDM. ${ }^{40}$

Retnakaran examined 851 women who underwent a glucose challenge test and a $3-\mathrm{h}$ oral glucose tolerance test in late pregnancy and pre-gravid PA. They reported that pre-gravid vigorous/sports activity is associated with a reduced risk of glucose intolerance in pregnancy. ${ }^{41}$
Luoto et al present a trial that was expected to be effective in the short-term prevention of gestational diabetes. The possible long-term benefits include prevention of chronic diseases, such as type 2 diabetes and cardiovascular diseases, both in the mother and offspring. ${ }^{6}$

Most of the intervention studies that have evaluated the role of PA in the prevention or treatment of glucose tolerance, excessive maternal weight gain and GDM, present protocols of intervention based on educational materials, individual counselling on diet or PA healthcare provider information or simply by administration of an interview questionnaire to collect information. 34121442

There are only a few studies in which all sessions of PA are developed in groups and supervised by a qualified fitness specialist; thus we found an important factor of novel and success of present study. In our opinion, pregnant women could exercise in an adequate, pleasant and amusing environment along with other pregnant women and this could generate an important level of adherence to the programme during the period of pregnancy.

Our results show benefits for EG women as they present minor values of $50 \mathrm{~g}$ MGS without potential risk for pregnancy outcome. We conclude that a moderate exercise programme during pregnancy improves the level of maternal glucose tolerance. In addition, we found a slight difference in maternal weight gain and cases of GDM, which could be viewed (exercise) as a protective factor. Our findings are promising given the increased active pregnant population. However, more RCTs are needed to further examine the efficacy of physical programmes on pregnancy outcome.

Funding This work was partially supported by the Ministerio de Ciencia e Innovación, Spain (DEP2009-08990).

\section{Competing interests None.}

Provenance and peer review Not commissioned; externally peer reviewed.

\section{REFERENCES}

1. American Diabetes Association. Gestational diabetes mellitus. Diabetes Care 2004;27:S88-S90.

2. Kaaja R, Rönnemaa T. Gestational diabetes: pathogenesis and consequences to mother and offspring. Rev Diabet Stud 2008;5:194-202.

3. Dempsey JC, Butler CL, Sorensen TK, et al. A case-control study of maternal recreational physical activity and risk of gestational diabetes mellitus. Diabetes Res Clin Pract 2004;66:203-15.

4. Chasan-Taber L, Marcus BH, Stanek E $3^{\text {rd }}$, et al. A randomized controlled trial of prenatal physical activity to prevent gestational diabetes: design and methods. $J$ Womens Health (Larchmt) 2009;18:851-9.

5. Ben-Haroush A, Yogev Y, Hod M. Epidemiology of gestational diabetes mellitus and its association with Type 2 diabetes. Diabet Med 2004;21:103-13.

6. Luoto RM, Kinnunen TI, Aittasalo M, et al. Prevention of gestational diabetes: design of a cluster-randomized controlled trial and one-year follow-up. BMC Pregnancy Childbirth 2010;10:39.

7. Mottola MF. The role of exercise in the prevention and treatment of gestational diabetes mellitus. Curr Diab Rep 2008;8:299-304.

8. Hedderson MM, Gunderson EP, Ferrara A. Gestational weight gain and risk of gestational diabetes mellitus. Obstet Gynecol 2010;115:597-604.

9. HAPO Study Cooperative Research Group. Hyperglycemia and adverse pregnancy outcomes. N Engl J Med 2008;358:1991-2002.

10. HAPO Study Cooperative Research Group. Hyperglycaemia and Adverse Pregnancy Outcome (HAPO) Study: associations with maternal body mass index. BJOG 2010:117:575-84.

11. Petry CJ. Gestational diabetes: risk factors and recent advances in its genetics and treatment. Br J Nutr 2010;104:775-87.

12. Polley BA, Wing RR, Sims CJ. Randomized controlled trial to prevent excessive weight gain in pregnant women. Int J Obes Relat Metab Disord 2002;26:1494-502.

13. Karagiannis T, Bekiari E, Manolopoulos K, et al. Gestational diabetes mellitus: why screen and how to diagnose. Hippokratia 2010;14:151-4.

14. Oken E, Ning Y, Rifas-Shiman SL, et al. Associations of physical activity and inactivity before and during pregnancy with glucose tolerance. Obstet Gynecol 2006;108:1200-7. 
15. American College of Sports Medicine; American Diabetes Association. Exercise and Type 2 Diabetes: American College of Sports Medicine and the American Diabetes Association: Joint Position Statement. Med Sci Sports Exerc 2010;42:2282-303

16. Davenport MH, Mottola MF, McManus R, et al. A walking intervention improves capillary glucose control in women with gestational diabetes mellitus: a pilot study. Appl Physiol Nutr Metab 2008;33:511-7.

17. Kim C. Gestational diabetes: risks, management, and treatment options. Int J Womens Health 2010;2:339-51.

18. Tobias DK, Zhang C, van Dam RM, et al. Physical activity before and during pregnancy and risk of gestational diabetes mellitus: a meta-analysis. Diabetes Care 2011:34:223-9.

19. Dempsey JC, Butler CL, Williams MA. No need for a pregnant pause: physical activity may reduce the occurrence of gestational diabetes mellitus and preeclampsia. Exerc Sport Sci Rev 2005;33:141-9.

20. Clapp JF. Effects of Diet and Exercise on Insulin Resistance during Pregnancy. Metab Syndr Relat Disord 2006;4:84-90.

21. Barakat R, Lucia A, Ruiz JR. Resistance exercise training during pregnancy and newborn's birth size: a randomised controlled trial. Int J Obes (Lond) 2009;33:1048-57.

22. Barakat R, Ruiz JR, Stirling JR, et al. Type of delivery is not affected by light resistance and toning exercise training during pregnancy: a randomized controlled trial. Am J Obstet Gynecol 2009;201:590.e1-6.

23. Barakat R, Ruiz JR, Rodríguez-Romo G, et al. Does exercise training during pregnancy influence fetal cardiovascular responses to an exercise stimulus? Insights from a randomised, controlled trial. Br J Sports Med 2010;44:762-4.

24. Moher D, Schulz KF, Altman D. The CONSORT statement: revised recommendations for improving the quality of reports of parallel-group randomized trials. JAMA 2001;285:1987-91.

25. ACOG. Exercise during pregnancy and the postpartum period. Committee Opinion $N^{0}$ 267. Washington, DC. January. Obstet Gynecol 2002;99:171-3.

26. Barakat R, Stirling JR, Lucia A. Does exercise training during pregnancy affect gestational age? A randomised controlled trial. Br J Sports Med 2008;42:674-8.

27. Bungum TJ, Peaslee DL, Jackson AW, et al. Exercise during pregnancy and type of delivery in nulliparae. J Obstet Gynecol Neonatal Nurs 2000;29:258-64.

28. Magann EF, Evans SF, Weitz B, et al. Antepartum, intrapartum, and neonata significance of exercise on healthy low-risk pregnant working women. Obstet Gynecol 2002;99:466-72
29. de Oliveira C, Lopes MA, Carla Longo e Pereira L, et al. Effects of pelvic floor muscle training during pregnancy. Clinics (Sao Paulo) 2007;62:439-46.

30. Dale E, Mullinax KM, Bryan DH. Exercise during pregnancy: effects on the fetus. Can J App/ Sport Sci 1982;7:98-103.

31. Cavalcante SR, Cecatti JG, Pereira RI, et al. Water aerobics II: maternal body composition and perinatal outcomes after a program for low risk pregnant women. Reprod Health 2009;6:1.

32. Bø K, Fleten C, Nystad W. Effect of antenatal pelvic floor muscle training on labor and birth. Obstet Gynecol 2009;113:1279-84.

33. de Barros MC, Lopes MA, Francisco RP, et al. Resistance exercise and glycemic control in women with gestational diabetes mellitus. Am J Obstet Gynecol 2010;203:556.e1-6.

34. Jovanovic-Peterson L, Peterson CM. Exercise and the nutritional management of diabetes during pregnancy. Obstet Gynecol Clin North Am 1996;23:75-86.

35. Jovanovic-Peterson L, Peterson CM. Is exercise safe or useful for gestational diabetic women? Diabetes 1991;40 Suppl 2:179-81.

36. Avery MD, Leon AS, Kopher RA. Effects of a partially home-based exercise program for women with gestational diabetes. Obstet Gynecol 1997;89:10-15.

37. Lesser KB, Gruppuso PA, Terry RB, et al. Exercise fails to improve postprandial glycemic excursion in women with gestational diabetes. J Matern Fetal Med 1996;5:211-17.

38. Dye TD, Knox KL, Artal R, et al. Physical activity, obesity, and diabetes in pregnancy. Am J Epidemiol 1997;146:961-5.

39. Bertolotto A, Volpe L, Calianno A, et al. Physical activity and dietary habits during pregnancy: effects on glucose tolerance. J Matern Fetal Neonatal Med 2010;23:1310-14.

40. Callaway LK, Colditz PB, Byrne NM, et al. Prevention of gestational diabetes: feasibility issues for an exercise intervention in obese pregnant women. Diabetes Care 2010;33:1457-9.

41. Retnakaran R, Qi Y, Sermer M, et al. Pre-gravid physical activity and reduced risk of glucose intolerance in pregnancy: the role of insulin sensitivity. Clin Endocrinol (Oxf) 2009;70:615-22.

42. Dempsey JC, Sorensen TK, Williams MA, et al. Prospective study of gestational diabetes mellitus risk in relation to maternal recreational physical activity before and during pregnancy. Am J Epidemiol 2004;159:663-70. 